\title{
Current and Emerging Antiarrhythmic Drug Therapy for Ventricular Tachycardia
}

Eric S. Williams • Mohan N. Viswanathan

To view enhanced content go to www.cardiologytherapy-open.com

Received: November 28, 2012 / Published online: February 20, 2013

(c) The Author(s) 2013. This article is published with open access at Springerlink.com

\section{ABSTRACT}

Ventricular arrhythmias, including ventricular fibrillation (VF) and sustained ventricular tachycardia (VT), are the principal causes of sudden cardiac death in patients with structural heart disease. While coronary artery disease is the predominant substrate associated with the development of VT, these arrhythmias are known to occur in a variety of disorders, including dilated cardiomyopathy, valvular and congenital heart disease, and cardiac ion channelopathies such as the long QT syndrome. In a minority of patients, VT occurs in the absence of structural heart disease. Despite the established mortality benefit of the implantable cardioverter defibrillator (ICD) in patients at risk of lethal arrhythmias, recurrent VT/VF

E. S. Williams $(\bowtie) \cdot$ M. N. Viswanathan Division of Cardiology, University of Washington Medical Center, Seattle, WA, USA

e-mail: ew49@uw.edu

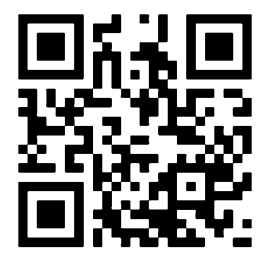

Enhanced content for this article is available on the journal web site: www.cardiologytherapy-open.com events continue to be a source of morbidity and impaired quality of life in such patients. Antiarrhythmic therapy is indicated in select patients to treat symptomatic VT episodes, to reduce the incidence of ICD shocks, and potentially to improve quality of life and reduce hospitalizations related to cardiac arrhythmia. The primary adverse effects of antiarrhythmic medications are related to both cardiac and extracardiac toxicity, including the risk of proarrhythmia. Current drug therapy for ventricular arrhythmia has been limited by suboptimal efficacy in many patients, resulting in recurrent VT/VF events, and by drug toxicity or intolerance leading to discontinuation in a large percentage of patients. Amiodarone and sotalol are the principal agents used in the chronic treatment of VT. In addition, dronedarone and dofetilide, agents approved for the treatment of atrial fibrillation, and ranolazine, an antianginal agent, have been demonstrated to be protective against ventricular arrhythmia in small clinical studies. Finally, advances in basic electrophysiology have uncovered new molecular targets for the treatment of ventricular arrhythmia, and pharmacologic agents directed at these targets 
may emerge as promising VT treatments in the future. The roles of these current and emerging therapies for the treatment of VT in humans will be summarized in this review.

Keywords: Antiarrhythmic medications; Ventricular fibrillation; Ventricular tachycardia

\section{INTRODUCTION}

Ventricular arrhythmias, including ventricular tachycardia (VT) and ventricular fibrillation (VF), are the leading cause of sudden cardiac death (SCD), which in turn represents about half of all cardiovascular mortality and accounts for over 350,000 deaths annually in the United States [1]. VT can be either sustained (lasting >30s) or nonsustained, and can have a uniform QRS morphology (monomorphic) or a variable morphology (polymorphic). It is the most common wide complex tachycardia seen in association with structural heart disease [2]. The vast majority of VT is related to myocardial pathologic processes that promote cardiac fibrosis or inflammation, most commonly from coronary artery disease (CAD) in over 80\% of patients [3]. However, myocarditis, dilated cardiomyopathy, congenital heart disease, cardiac infiltrative diseases, arrhythmogenic right ventricular cardiomyopathy, and hypertrophic cardiomyopathy are also known to contribute to an arrhythmogenic substrate. In about $10 \%$ of patients, VT occurs in the absence of structural heart disease [4]. This subset of VT is thought to be either idiopathic or related to primary electrical disease, such as the long QT syndrome, Brugada syndrome, catecholaminergic polymorphic ventricular tachycardia (CPVT), or other cardiac ion channelopathies $[5,6]$.
The principal mechanisms of arrhythmogenesis in the heart are abnormal automaticity, triggered activity from afterdepolarizations, and myocardial reentry [2]. Triggered activity and abnormal automaticity are the most important mechanisms of focal VT arising from the ventricular outflow tracts, although microreentrant circuits may also play a role. In the setting of myocardial scar from CAD, macroreentry is the most common mechanism contributing to VT $[7,8]$. It is estimated that $1-5 \%$ of all patients with a history of previous myocardial infarction (MI) will develop VT. In the setting of acute MI, on the other hand, the incidence of VT/VF ranges from $2 \%$ to $10 \%[9,10]$. This upfront arrhythmic risk has been reduced by early coronary reperfusion strategies, such as thrombolytics and primary angioplasty, in the acute phases of MI [2, 11]. The electrical substrate for VT following acute $\mathrm{MI}$ is established as early as 2 weeks postinfarction, based on programmed ventricular stimulation studies, and presumably is present indefinitely [12]. The abnormal substrate is characterized by inflammation and fibrosis, cardiac hypertrophy, abnormal cell coupling, and ion channel expression in the myocardium that promote ventricular arrhythmia [5]. The subsequent development of VT in at-risk patients results from the interplay of the abnormal myocardial substrate and arrhythmogenic triggers. The roles played by the autonomic nervous system, hemodynamic stress, metabolic abnormalities, and ventricular premature depolarizations as proarrhythmic triggers have all been well described $[6,13]$.

The only intervention demonstrated to improve survival in patients at risk of SCD from ventricular arrhythmias is the implantable cardioverter defibrillator (ICD). It is indicated for secondary prevention in patients with a 
history of sustained VT/VF, and for primary prevention in patients with a history of heart failure or previous $\mathrm{MI}$ and left ventricular ejection fraction (LVEF) of $35 \%$ or less [14]. There are several limitations, however, with the ICD as primary therapy for VT/VF. First, and most important, is that although the ICD effectively terminates ventricular arrhythmias, it does not prevent them. Second is the morbidity associated with both appropriate and inappropriate ICD shocks. Third, the current selection criteria for ICD candidacy are imperfect, as many ICD recipients never receive appropriate ICD therapy for VT/VF, whereas many other patients with LVEF greater than $35 \%$ who are not eligible for the ICD go on to experience SCD [15]. In addition, the benefit of the ICD is not established in the early post-MI period; despite an increased risk of arrhythmic death in this population, there was no difference in total mortality in patients within 6 and 40 days of acute MI treated with the ICD vs. medical therapy in a randomized trial [16].

Antiarrhythmic drug therapy is commonly used as adjunctive treatment in ICD recipients for the suppression of VT/VF episodes. In the Antiarrhythmic Versus Implantable Defibrillator (AVID) trial of secondary prevention ICD therapy, the 1-year arrhythmia event rate was $90 \%$ in the ICD arm, and was reduced to $64 \%$ with concurrent antiarrhythmic therapy [17]. Overall, up to $70 \%$ of patients with an ICD receive adjuvant antiarrhythmic drug therapy, even though there is no medication formally approved for this indication [18]. The indications for adjunctive antiarrhythmic therapy are: to reduce the incidence of appropriate and inappropriate ICD shocks; to slow the rate of spontaneous VT episodes to improve their hemodynamic tolerance and to facilitate pace termination by the ICD; to treat symptomatic VT episodes; to improve quality of life; and potentially to reduce hospitalizations related to cardiac arrhythmia [18].

Current antiarrhythmic therapy for VT is limited by its potential for both cardiac and extracardiac toxicity, including the risk of proarrhythmia, and by its limited efficacy. In the Optimal Pharmacological Therapy in Cardioverter Defibrillator Patients (OPTIC) trial, amiodarone and sotalol were each significantly more effective in preventing ICD shocks compared to beta-blockers alone, but 1 -year shock rates were still $10 \%$ in the amiodarone arm and 24\% in the sotalol arm, with drug-related adverse effects leading to discontinuation in one in five patients [19]. No new antiarrhythmic agents have yet been approved for the treatment for VT in the past decade; however, novel concepts in the understanding of ventricular arrhythmogenesis have the potential to deliver new therapeutic targets for VT that balance antiarrhythmic efficacy against the risks of organ toxicity, negative inotropy, and proarrhythmic effects seen with contemporary drug therapy.

Several clinical trials have evaluated the efficacy and safety of various antiarrhythmic medications used for the treatment of VT in patients with established cardiovascular disease. This review will summarize their findings and discuss more recent data on emerging pharmacotherapies for ventricular arrhythmia.

\section{METHODS}

The following review article incorporates data from clinical trials, review articles, and textbooks to provide a comprehensive and upto-date summary of antiarrhythmic drug therapy for VT. Emerging antiarrhythmic therapies include those agents that have not yet been approved for clinical use in VT but 
have been tested in clinical investigations or early phase clinical trials in humans in the past decade (2002-2012).

\section{DISCUSSION}

\section{Current Antiarrhythmic Therapy}

\section{Classification of Antiarrhythmic Agents}

The most common classification scheme for antiarrhythmic agents is the Vaughan Williams classification, which characterizes drugs based on their ability to block specific ion currents or cell receptors [20]. Table 1 summarizes these agents and their use in the treatment of ventricular arrhythmias, and Table 2 summarizes the results of select clinical trials with these medications. Class I agents are sodium channel blockers, further divided into Class IA (quinidine, procainamide and disopyramide), Class IB (lidocaine, mexiletine), and Class IC (flecainide, propafenone). Class II agents are beta-adrenergic receptor blockers, such as propranolol. Class III agents are potassium channel blockers, such as amiodarone, sotalol, dofetilide, and dronedarone. Class IV agents are calcium channel blockers, such as verapamil. The Vaughan Williams classification does not, however, account for the complex actions of certain antiarrhythmics, such as amiodarone, which is known to have multichannel blocking properties [38].

\section{Beta-Blockers}

Beta-blockers are considered first-line therapy for patients with systolic heart failure and following acute MI for their established survival benefit in these populations [30, 31, $39,40]$. In addition, beta-blockers are indicated in the treatment of certain ion channelopathies, such as congenital long QT syndrome and CPVT [41].

In the Cardiac Insufficiency Bisoprolol Study II (CIBIS-II), bisoprolol reduced all-cause mortality by $34 \%$ and sudden cardiac death by $44 \%$ in patients with heart failure [30]. The Clopidogrel and Metoprolol in Myocardial Infarction Trial (COMMIT) randomly assigned over 45,000 patients to either a combination of intravenous and oral metoprolol or placebo within $24 \mathrm{~h}$ of acute MI, and showed that the use of early beta-blocker therapy reduced the risk of VF development, although this was counterbalanced by an increase in cardiogenic shock, especially during the first day after admission [31]. Overall, a meta-analysis of beta-blocker studies in post-MI patients suggests a significant relative benefit in preventing SCD and all-cause mortality [42].

\section{Amiodarone}

Amiodarone is an iodinated benzofuran derivative that is highly lipophilic. It combines properties of all Vaughan Williams classifications, possessing sodium channel, potassium channel, calcium channel, and beta-adrenergic receptor blocking activity. It accumulates in a variety of organ tissues, including adipose, leading to an elimination half-life of over 30 days. In fact, amiodarone can be detected in plasma up to 9 months after discontinuation [22].

In the pre-ICD era, amiodarone had an established use for the prevention of SCD in high-risk patients with a history of previous MI or aborted SCD [43]. Meta-analysis showed a modest reduction in all-cause mortality with amiodarone vs. placebo [44]. A pooled analysis of the European Amiodarone Myocardial Infarction Trial (EMIAT) and the Canadian Amiodarone Myocardial Infarction Trial (CAMIAT) that evaluated amiodarone use in 


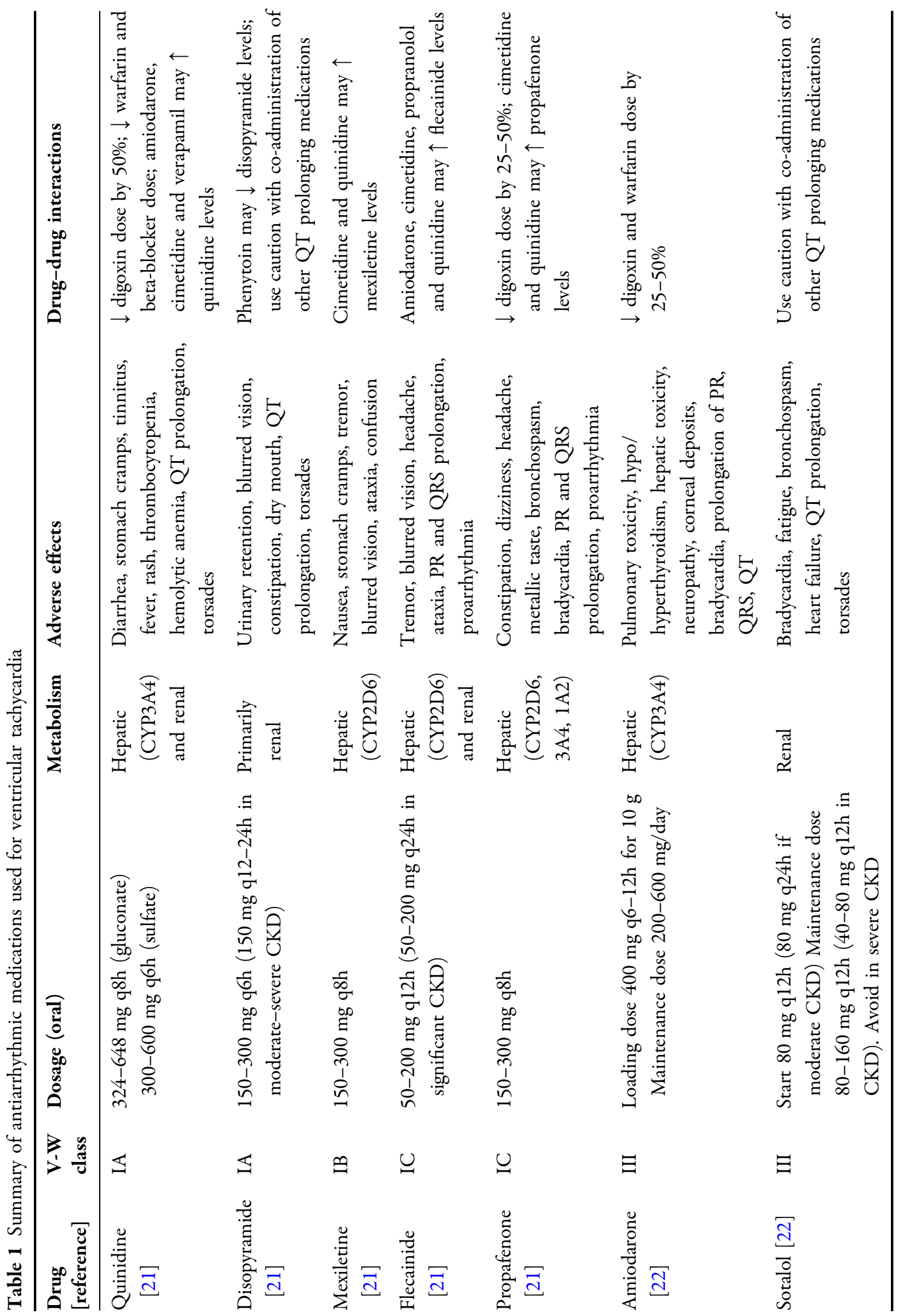




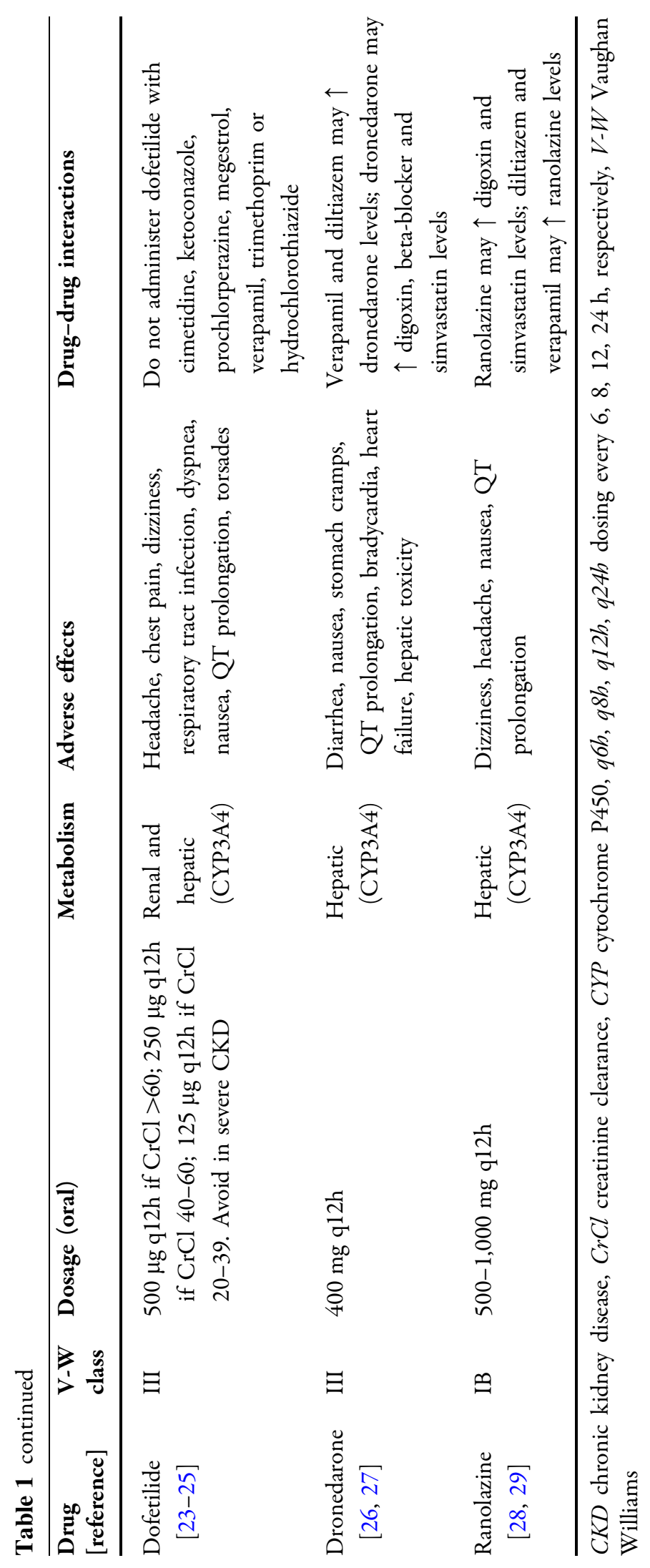




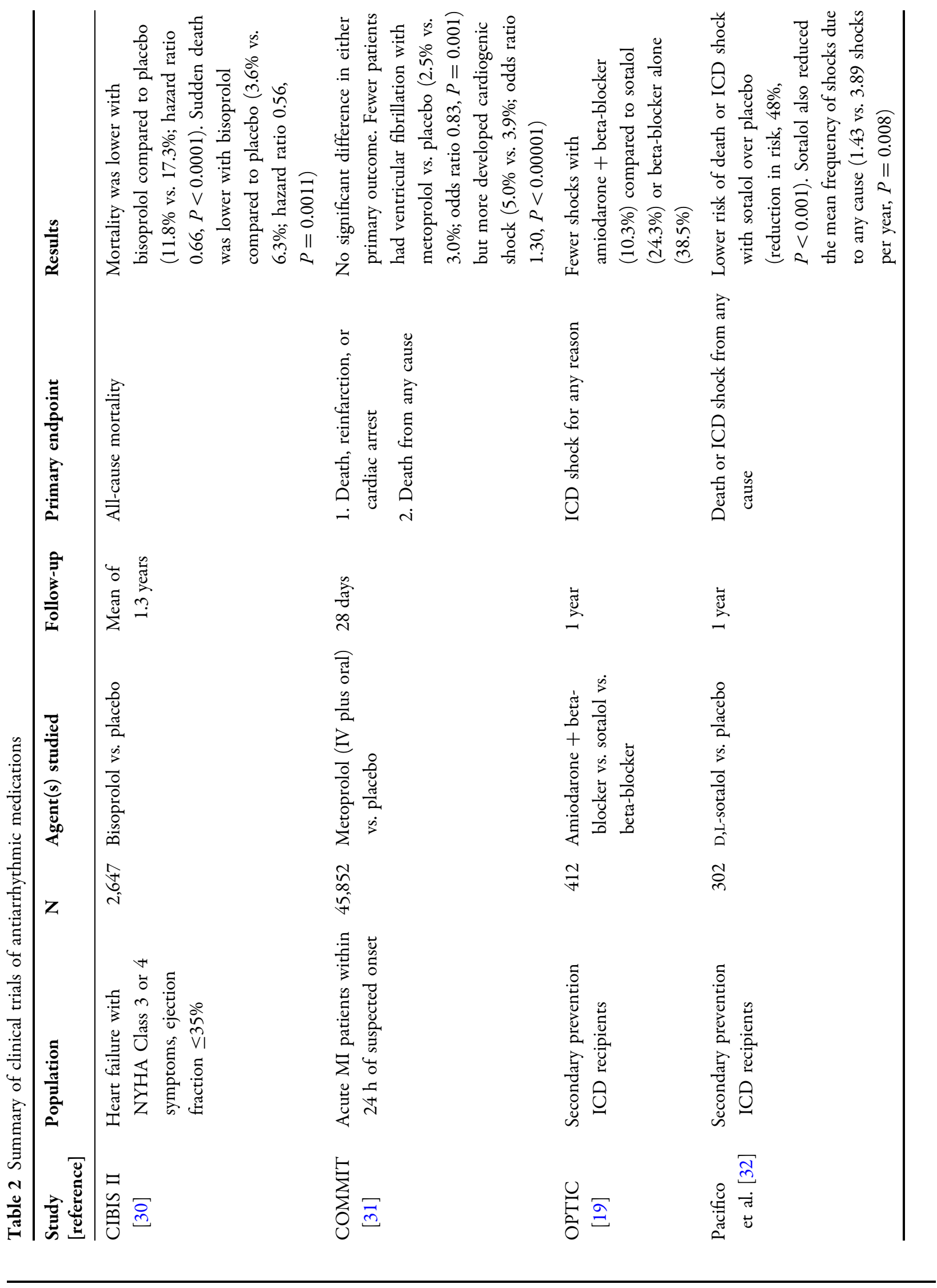




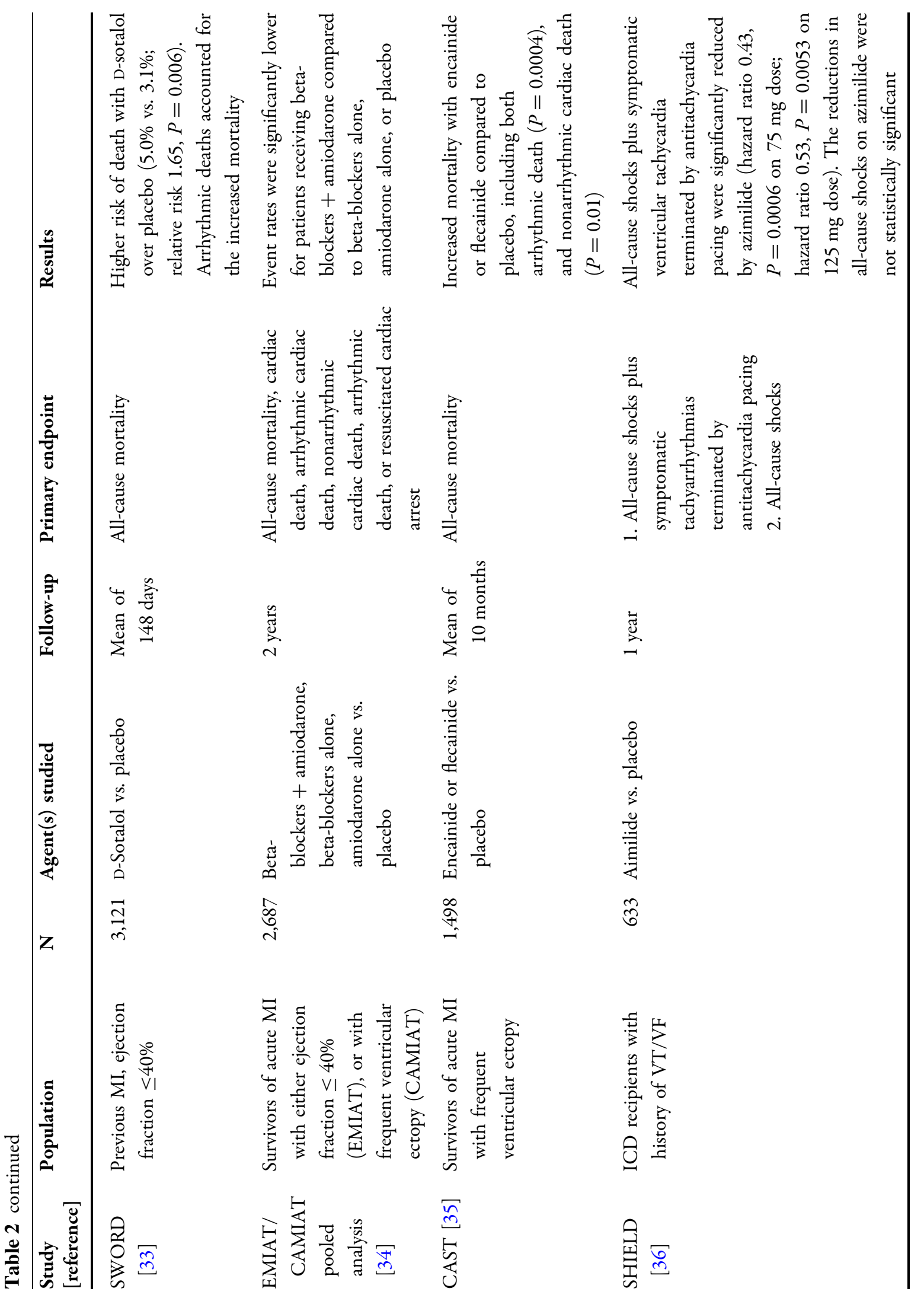




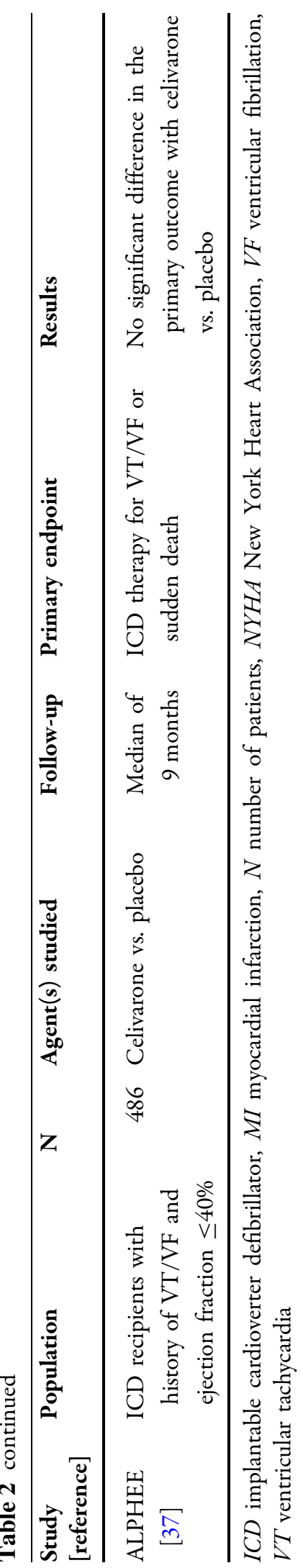

patients recovering from MI (EMIAT enrolled patients with LVEF $\leq 40 \%$ and CAMIAT enrolled patients with frequent or repetitive ventricular ectopy) found that incidences of cardiovascular death and arrhythmic death or resuscitated cardiac arrest were significantly lower in patients receiving both beta-blockers and amiodarone than in those not receiving betablockers, with or without amiodarone [34]. Conversely, in the era of the primary prevention ICD, amiodarone did not confer a survival benefit over placebo in the Sudden Cardiac Death in Heart Failure Trial (SCD-HeFT) of symptomatic heart failure patients with LVEF of $35 \%$ or less [45].

Amiodarone plus beta-blockers was shown to be superior to monotherapy with sotalol or beta-blockers in the OPTIC trial for the reduction of shocks in secondary prevention ICD recipients [19]. As a result of its greater efficacy, amiodarone is the most common antiarrhythmic agent used for suppression of VT in patients with structural heart disease and ICDs. In the AVID trial, nearly twice as many patients in the ICD arm who ultimately received adjuvant antiarrhythmic therapy were treated with amiodarone compared to either sotalol or mexiletine [17].

Amiodarone has a low risk of proarrhythmia, despite causing prolongation of the action potential duration and QT interval, probably because it reduces heterogeneity of depolarization. Torsade de pointes occurred in less than $1 \%$ in the EMIAT and CAMIAT trials $[46,47]$. Extracardiac toxicity, however, is well described, and is related to both a daily and cumulative dose effect of amiodarone. Clinical hypothyroidism occurs in up to $32 \%$ of patients, and may require thyroxine supplementation even after drug discontinuation [22]. Hyperthyroidism can also occur, but is less common in the western world where dietary 
iodine intake is adequate. Pulmonary toxicity is less common but is among the most serious adverse drug reactions, presenting as chronic interstitial pneumonitis, bronchiolitis obliterans with organizing pneumonia, or the acute respiratory distress syndrome. Corneal deposits, skin photosensitivity, neuropathy, and gastrointestinal side effects have also been reported [22].

\section{Sotalol}

Sotalol is a potassium channel blocker that prolongs action potential duration and is a Vaughan Williams Class III agent. It is a racemic mixture of D-sotalol, which has pure Class III antiarrhythmic activity, and L-sotalol, which has Class III and beta-blocker effects. Doses less than $120 \mathrm{mg}$ twice daily appear to have a primary beta-blocker effect, with higher doses producing significant Class III activity [22].

A placebo-controlled trial in 302 ICD recipients showed that treatment with racemic sotalol significantly reduced the risk of death or ICD shock (34\% incidence with sotalol vs. $54 \%$ with placebo) at 1 year [32]. However, the rate of drug discontinuation in the sotalol arm was $27 \%$. A similar finding was noted in the OPTIC trial, with nearly a quarter of patients discontinuing sotalol therapy due to drug intolerance [19]. The most common adverse reactions in these trials were related to the beta-blocking effects of the drug; symptomatic bradycardia and torsade de pointes were rare. Of note, in the Survival With Oral D-Sotalol (SWORD) trial, D-sotalol, which does not have significant beta-blocking effects, was associated with increased mortality and proarrhythmia in patients with post-MI left ventricular dysfunction [33].

The most significant adverse reaction associated with sotalol is torsade de pointes, seen in $2-3 \%$ of patients; especially at risk are women and patients with heart failure or chronic kidney disease (because of its significant renal drug elimination) [48]. For this reason, it is common practice to initiate sotalol therapy in the inpatient setting with continuous ECG monitoring during the loading phase for five doses in patients at higher risk. QT interval prolongation and bradycardia can presage the development of proarrhythmia and may warrant a reduction of the sotalol dose. Other adverse effects include fatigue, bronchospasm, dyspnea and heart failure. Unlike amiodarone, these effects are related to the daily dose but not the cumulative dose, making sotalol a more attractive first-line therapy for younger patients or those for whom longer-term treatment is anticipated [22].

\section{Class I Antiarrhythmic Agents}

The Cardiac Arrhythmia Suppression Trial (CAST) compared Class IC agents to placebo in post-MI patients with impaired LVEF (40\% or less) for the suppression of ventricular ectopy, and was terminated prematurely due to excess mortality in the antiarrhythmic arm [35]. Both all-cause mortality and arrhythmic death were increased with both encainide and flecainide treatment. As such, Class IC antiarrhythmic agents are no longer recommended therapy for patients with ischemic heart disease or left ventricular dysfunction from any cause. Conversely, the risk of ventricular proarrhythmia with Class IC agents in the absence of structural heart disease is low; however, in patients with atrial arrhythmias, flecainide or propafenone may promote 1:1 atrioventricular nodal conduction with acceleration of the ventricular rate and a wide QRS tachycardia [21].

Earlier studies that examined Class I agents for secondary VT/VF prevention in post-MI patients showed they were inferior in efficacy to both amiodarone and sotalol $[49,50]$. The 
most commonly used Class I agent in this setting is mexiletine, used in $20 \%$ of patients who received adjuvant antiarrhythmic treatment in the ICD arm of the AVID trial [17]. As a Class IB antiarrhythmic agent, it does not seem to carry the increased mortality risk associated with the Class IC drugs, based on observational data with the Class IB drug lidocaine from the Global Utilization of Streptokinase and TPA for Occluded Coronary Arteries (GUSTO-I and GUSTO-IIb) trials [51].

Quinidine, procainamide, and disopyramide are Class IA antiarrhythmic agents that have intermediate sodium channel blocker activity (compared to Class IC agents) and also prolong action potential duration via potassium channel blockade. They are indicated in the treatment of supraventricular arrhythmias and VT. Unfortunately, use of these agents is limited by the risk of torsade de pointes (seen in $0.5-8 \%$ ) and the poor tolerability of these agents, including drug-induced lupus with procainamide, anticholinergic effects with disopyramide, and a host of gastrointestinal, dermatologic and neurologic side effects seen with quinidine use [21].

While the lower efficacy and poor tolerability of the Class I agents has relegated them to third-line therapy for the prevention and treatment of ventricular arrhythmia, there is evidence that combination therapy with a Class I and a Class III agent may be more effective than monotherapy with either agent [43]. Common combinations include amiodarone or sotalol plus mexiletine.

\section{Emerging Antiarrhythmic Therapy}

\section{Dronedarone}

Dronedarone is a recent addition to the antiarrhythmic armamentarium. A Vaughan Williams Class III agent, dronedarone is a multichannel blocker similar in structure to amiodarone but noniodinated. It was developed with the potential to achieve antiarrhythmic efficacy similar to that of amiodarone, without the extracardiac toxicity seen with long-term amiodarone therapy [26, 52]. It is approved for the treatment of atrial fibrillation, largely based on results of A Trial With Dronedarone to Prevent Hospitalization or Death in Patients With Atrial Fibrillation (ATHENA), a placebo-controlled, double-blind, parallel arm trial to assess the efficacy of dronedarone $400 \mathrm{mg}$ b.i.d. for the prevention of cardiovascular hospitalization or death from any cause in patients with atrial fibrillation or atrial flutter, which demonstrated significant reductions in the composite endpoint of all-cause mortality and cardiovascular hospitalization with dronedarone vs. placebo [26]. In two earlier randomized trials of dronedarone in patients with atrial fibrillation or flutter, rates of pulmonary, thyroid, and hepatic adverse effects were not significantly greater with dronedarone than with placebo at 1 year follow-up [27]. After its approval in the United States, however, subsequent reports of severe liver toxicity led to a warning by the US Food and Drug Administration, recommending that prescribing physicians follow hepatic function tests routinely [53].

Although dronedarone has not been studied specifically for the treatment of VT/VF, animal studies have demonstrated antiarrhythmic properties on ventricular myocardium, and subsequent reports in humans have supported its efficacy in select cases [54-56]. In addition, in ATHENA, patients on dronedarone showed a reduction in arrhythmic death [26]. The use of dronedarone in patients with heart failure, however, is controversial in light of the Antiarrhythmic Trial with Dronedarone in Moderate to Severe CHF Evaluating Morbidity 
Decrease (ANDROMEDA) trial, whose results suggest dronedarone may lead to worsening heart failure symptoms and a two-fold increase in mortality in this population [52]. As such, dronedarone is contraindicated in Class IV heart failure patients or in those who have had a recent hospitalization for decompensated heart failure. The ANDROMEDA study authors recommend that "dronedarone should not be used in patients with heart failure and reduced left ventricular systolic function." A more recent placebocontrolled trial of dronedarone in patients with permanent atrial fibrillation and major vascular risk factors (including $\mathrm{CAD}$ and heart failure) was stopped prematurely due to a two-fold excess in cardiovascular mortality [57]. Stroke, hospitalization for heart failure, and arrhythmic deaths were also significantly increased in the dronedarone arm of the Permanent Atrial Fibrillation Outcome Study Using Dronedarone on Top of Standard Therapy (PALLAS) [57]. While some of these adverse findings were unexplained, it was postulated that the negative inotropic effects of dronedarone, along with its drug-drug interactions (notably with vitamin $\mathrm{K}$ antagonists and with digoxin) and potential proarrhythmic effects, may have contributed.

In summary, while dronedarone has been shown to be effective in suppressing ventricular arrhythmia in animal studies and in case reports of patients with refractory VT/VF episodes, the results of ANDROMEDA and PALLAS have raised doubts about the safety of this medication in patients with structural heart disease.

\section{Dofetilide}

Dofetilide is a Class III antiarrhythmic agent and a selective blocker of the rapid delayed rectifier potassium current, $\mathrm{I}_{\mathrm{Kr}}$ [58]. It is approved in North America for the treatment of atrial fibrillation; however, it has been shown to have efficacy in the treatment of ventricular arrhythmia. A randomized trial of patients with CAD and sustained VT showed that oral dofetilide was equally as effective as oral sotalol in the prevention of recurrent ventricular arrhythmias and arrhythmic death at 1 year [59]. A more recent study in 30 ICD recipients with drug-refractory VT/VF episodes showed a significant reduction in both monthly ventricular arrhythmia episodes (from $1.8 \pm 4.5$ to $1.0 \pm 3.5, P=0.006$ ) and monthly ICD therapies (from $0.9 \pm 1.4$ to $0.4 \pm 1.7$, $P=0.037)$ after treatment with dofetilide. In addition, $83 \%$ of patients had complete suppression of VT/VF during their first month of treatment [60].

Dofetilide is very well tolerated, although inpatient monitoring for 3 days is required during the loading phase, given the risk of QT prolongation and the potential for torsade de pointes (seen in 1-3\%) [23, 24]. Dofetilide dosing is based on calculated creatinine clearance, as a result of its renal drug elimination. The safety of dofetilide has been established in patients with left ventricular dysfunction and CAD [24, 25], and on the basis of limited clinical experience in the treatment of ventricular arrhythmia, it may be an alternative antiarrhythmic agent for such patients with VT/VF events refractory to amiodarone and/or sotalol therapy.

\section{Ranolazine}

Ranolazine is a novel antianginal drug with multiple ion channel blocking antiarrhythmic activity. It is a piperazine derivative with a chemical structure similar to lidocaine, and its most potent ion channel blocking effect is on late sodium current $[28,29,61,62]$. It is thus considered a Vaughan Williams Class IB agent. Ranolazine also has effects on the delayed rectifier current $\left(\mathrm{I}_{\mathrm{Kr}}\right)$ and prolongs action 
potential duration, with corresponding QT interval prolongation on electrocardiography. It has been shown in experimental animal models to have antiarrhythmic effects in the ventricle [61, 62]. In the Metabolic Efficiency With Ranolazine for Less Ischemia in Non-ST-Elevation Acute Coronary SyndromeThrombolysis in Myocardial Infarction 36 trial (MERLIN-TIMI 36), ranolazine was shown clinically to reduce arrhythmia episodes, including nonsustained VT, on ambulatory cardiac monitoring in patients presenting with acute coronary syndrome [29]. It has subsequently been used in the suppression of ectopic ventricular activity [63] and for the reduction in VT burden and prevention of shocks in ICD recipients [64].

Ranolazine in particular works synergistically with the Class III antiarrhythmic agents, most commonly with amiodarone [65]. This has been demonstrated in animal models to have an antiarrhythmic effect in both the atrium and ventricle. In rabbit hearts treated with both ranolazine and a Class III agent, there was no increase in early after-depolarizations or ventricular proarrhythmia associated with the addition of ranolazine [61]. In addition, in the MERLIN-TIMI 36 trial, despite causing modest QT prolongation, ranolazine use was not associated with an increased risk of SCD compared with placebo [66]. Based on limited but positive clinical experiences with ranolazine, it appears to be beneficial as add-on therapy in patients with recurrent VT events while on a Class III antiarrhythmic agent.

\section{Azilimide}

Azimilide is an investigational Class III antiarrhythmic agent that blocks both the rapid $\left(\mathrm{I}_{\mathrm{Kr}}\right)$ and slow $\left(\mathrm{I}_{\mathrm{Ks}}\right)$ components of the delayed rectifier cardiac potassium current. It causes prolongation of the atrial and ventricular action potential duration and refractory period [36]. As such, azimilide has demonstrated action against both supraventricular and ventricular arrhythmias. In the Shock Inhibition Evaluation with Azimilide (SHIELD) trial, a randomized controlled trial of 633 secondary prevention ICD recipients, the primary endpoint of all-cause shocks plus symptomatic tachyarrhythmias terminated by antitachycardia pacing was significantly reduced in patients receiving azimilide [36]. In addition, the secondary endpoint of appropriate ICD therapies for VT/VF episodes was reduced by $48 \%$ and $62 \%$, with the $75 \mathrm{mg}$ and $125 \mathrm{mg}$ doses of azimilide, respectively.

Based on the concerning results from previous antiarrhythmic drug trials in patients with structural heart disease, such as CAST and SWORD, azimilide was studied prospectively in the Azimilide Postinfarct Survival Evaluation (ALIVE) trial, in which 3,717 patients with recent $\mathrm{MI}$ and an ejection fraction between $15 \%$ and $35 \%$ were randomly assigned to receive azimilide, $100 \mathrm{mg}$ daily, vs. placebo. At 1 year of follow-up, there were no significant differences in all-cause, cardiac, or arrhythmic mortality between the azimilide and placebo groups [67].

Overall, azimilide was well tolerated in clinical trials. In the SHIELD trial, its discontinuation rate was similar to the placebo arm. Adverse events with azimilide include neutropenia (seen in 1\% of patients) and QT prolongation leading to torsade de pointes (seen in up to $1-2 \%$ of patients). It is not currently approved for use in North America or Europe.

\section{Celivarone}

Celivarone is a noniodinated benzofuran derivative that is in investigational use for its action against atrial and ventricular arrhythmias [37]. Similar to amiodarone and 
dronedarone, it has Class I, II, III and IV antiarrhythmic activity, but with different relative potencies for the various channels and receptors. Also, its structure and kinetics differ from those of amiodarone and lend itself to an improved side effect profile and reduced potential for drug interactions [68]. It was shown in a small phase 2 clinical study of ICD recipients to trend toward fewer VT and VF episodes at the higher dose of celivarone (300 mg daily), although the $46 \%$ relative risk reduction at 6 months was not statistically significant [69]. A larger trial of 486 patients with LVEF of $40 \%$ or less and at least one VT/VF episode within a month of enrollment, however, did not find that celivarone was any more effective for the prevention of ICD interventions or sudden death than placebo [68]. In both studies, celivarone was well tolerated and had an acceptable safety profile. Nonetheless, in light of the disappointing clinical data to date, it is not currently approved for use in humans.

\section{Future Antiarrhythmic Targets}

Novel targets for the treatment of ventricular arrhythmia continue to be explored, and it is likely that pharmacologic agents directed at some of these targets will enter clinical trials in the next few years. The commonly used antiarrhythmic medications for VT/VF primarily target sodium channels (Class I agents) or potassium channels (Class III agents), but are limited by variable efficacy and the potential for ventricular proarrhythmia. Newer therapeutic approaches to cardiac arrhythmias have focused on the roles of intracellular calcium, gap junctions, sodium-calcium exchange, and adenosine triphosphate (ATP)-sensitive potassium channel blockade, and will be reviewed briefly [37].

\section{Intracellular Calcium}

Altered intracellular calcium handling has been implicated in ventricular arrhythmogenesis in a number of models [6]. Two important proteins in myocardial calcium homeostasis are the sarcoplasmic reticulum (SR) calcium ATPase (SERCA2a) and the ryanodine receptor (RyR2). The former promotes calcium reuptake into the SR and the latter is a SR calcium release channel that promotes an increase in cytosolic calcium, which in turn activates myocardial contractile proteins. Diastolic calcium leakage via RyR2 is thought to contribute to proarrhythmia, notably by promoting after-depolarizations in the cardiomyocyte. CPVT is one cardiac electrical disorder characterized by leaky RyR2, resulting in delayed after-depolarizations and polymorphic VT triggered by exercise and adrenergic stimulation $[6,41]$. The antiarrhythmic agent flecainide targets RyR2, and was shown to prevent arrhythmias in a mouse model of CPVT, by inhibiting RyR2mediated calcium release. Now this agent has found a role clinically to suppress VT events in patients with CPVT in conjunction with betablockers [70].

Pharmacotherapies to normalize intracellular calcium handling by either stabilizing RyR2 activity or modulating associated proteins involved in diastolic SR calcium leakage in order to prevent arrhythmia may prove to be novel antiarrhythmic agents in the future. In a recent report, a pharmacologic RyR2 stabilizer was investigated in both a mouse model and in human nonfailing myocardium, and was found to be effective in reducing SR calcium leak [71]. Another recent report showed that inhibition of calcium/calmodulin-dependent kinase (CaMKII) was able to reduce cardiac arrhythmias and SCD in a proarrhythmic mouse model similar to that seen in CPVT [72]. 


\section{Gap Junctions}

Cell-cell coupling in the heart acts to maintain synchronization of depolarization and repolarization between myocytes, and disruption of this coupling is thought to contribute to arrhythmogenesis. It has been proposed that restoration or enhancement of coupling via gap junctions may be an effective antiarrhythmic target [37]. Connexin 43 is the principal gap junction protein responsible for cell-cell coupling in ventricular myocardium, and its function is impaired during acute ischemia and acidosis [73]. Rotigaptide, an antiarrhythmic peptide that improves conduction across gap junctions, has been shown in experimental animal models to suppress ischemia-induced proarrhythmia [73]. The proposed mechanism of action of rotigaptide is prevention of the dephosphorylation of connexin 43 that accompanies acute metabolic stress. By maintaining gap junction conductance, this peptide in turn both prevents conduction slowing in the cardiomyocytes, and synchronizes the action potentials thereby reducing dispersion of refractoriness [74].

While the concept of normalizing gap junction conductance with an antiarrhythmic agent is a promising one, there are multiple mechanisms by which gap junction physiology can be impaired in disease states other than by dephosphorylation. The roles of myocyte fibrosis, connexin protein downregulation and trafficking in the remodeling of gap junctions have all been appreciated and may pose challenges to the development of a single pharmacotherapeutic target or agent [73].

\section{Sodium-Calcium Exchange}

The sodium-calcium exchanger (NCX) is the primary pathway for intracellular calcium removal in the cardiomyocyte. It is a cell membrane protein that removes a single calcium ion in exchange for the import of three sodium ions, while operating in the forward mode. Increased expression or activity of NCX has been associated with impaired cardiac contractility and an increased risk of arrhythmias in the setting of heart failure [75]. NCX also operates in the reverse mode, promoting intracellular calcium loading, during conditions of high cytosolic sodium concentration, or in the setting of digitalis use (which antagonizes the sodium/potassium ATPase). Excessive calcium loading can also be proarrhythmic, as it promotes triggered activity through delayed after-depolarizations [6, 75].

NCX blockade has been considered to be a potential therapeutic strategy for cardiac arrhythmias, in particular with agents that predominantly inhibit the reverse mode over the forward mode. To date, there has been limited progress in the development of clinically useful agents. Two drugs, KBR-7943 and SEA-0400, have been shown to prevent calcium overload in models of ischemia/reperfusion injury, and appear to reduce after-depolarizations in models of vulnerable cardiac tissue [75]. These findings are promising but await further in vivo confirmation in animal models.

\section{ATP-Sensitive Potassium Channel Blockade}

Myocardial ischemia is associated with increases in extracellular potassium, which is believed to contribute to ventricular proarrhythmia. The activation of cardiac cell membrane ATPsensitive potassium channels during myocardial ischemia promotes potassium efflux and reductions in action potential duration; impaired function of the sodium/potassium ATPase may also contribute [76]. In addition, ischemia-induced potassium accumulation is heterogeneous, which leads to dispersion of repolarization and thereby creates a substrate for reentrant arrhythmias. 
ATP-sensitive potassium channel activity is inhibited by ATP but activated by adenosine $5^{\prime}$ diphosphate (ADP). Therefore, with a fall in the ATP:ADP ratio during myocardial ischemia, the ATP-sensitive potassium channel opens and potassium leaves the cell. Increases in extracellular potassium are known to promote perturbations in cardiac electrical activity, such as increased excitability of normal ventricular tissues, leading to premature ventricular complexes, and a reduction in action potential duration. Regional dispersion of the refractory period, especially during periods of myocardial ischemia, is a major contributor to the development of VF. Glibenclamide is an ATPsensitive potassium channel inhibitor that has been shown to attenuate reductions in action potential duration in models of ischemia, and suppress extrasystoles and VF [76].

Glibenclamide is a sulfonylurea that also provokes hypoglycemia due to its effects on noncardiac tissue [76]. For ATP-sensitive potassium channel inhibition to become an attractive therapeutic option, cardioselective pharmaceuticals must be developed and tested. Currently, the agents HMR-1883, HMR1098 and HMR-1402 have been developed and studied in animals, with favorable results on the reduction of ischemic cardiac arrhythmias [77].

\section{CONCLUSION}

In patients with structural heart disease at risk of ventricular arrhythmias, the ICD continues to be the gold standard therapy for the reduction of SCD and improved long-term survival. Antiarrhythmic medication is indicated as addon therapy in those with VT events to reduce the morbidity associated with recurrent arrhythmic episodes. While amiodarone and sotalol are the principal agents used in this setting, their use is often limited by suboptimal effectiveness and drug intolerance or toxicity leading to discontinuation.

Newer and emerging antiarrhythmic therapies must meet the challenge of effectively suppressing drug-refractory VT/VF without promoting proarrhythmia or other cardiovascular adverse events. While dofetilide and ranolazine hold promise, and merit further investigation in large prospective studies of ICD patients, dronedarone use in atrial fibrillation has been associated with a disturbing signal of harm in patients with structural heart disease. Therefore its use in patients with VT should be carefully considered or avoided in the absence of prospective data establishing its safety and efficacy in this population. Azimilide and celivarone await more convincing efficacy data in humans before they are approved for clinical use.

A better understanding of the molecular mechanisms of ventricular arrhythmogenesis has provided basic electrophysiologists with new antiarrhythmic targets related to intracellular calcium handling, gap junctions, sodium-calcium exchange, and ATP-sensitive potassium channel activity. Further advances in this field will undoubtedly spur novel drug therapies for patients with refractory ventricular arrhythmias in the future.

\section{ACKNOWLEDGMENTS}

Dr. Williams is the guarantor for this article, and takes responsibility for the integrity of the work as a whole. No extramural funding was used in the preparation of this manuscript.

Conflict of interest. Dr. Williams declares he has no conflicts of interest. Dr. Viswanathan declares he has no conflicts of interest. 
Open Access. This article is distributed under the terms of the Creative Commons Attribution Noncommercial License which permits any noncommercial use, distribution, and reproduction in any medium, provided the original author(s) and the source are credited.

\section{REFERENCES}

1. Roger VL, Go AS, Lloyd-Jones DM, et al. Executive summary: heart disease and stroke statistics-2012 update: a report from the American Heart Association. Circulation. 2012;125:188-97.

2. Josephson ME. Recurrent ventricular tachycardia. In: Clinical cardiac electrophysiology: techniques and interpretations. 4th ed. Philadelphia: Lippincott Williams \& Wilkins; 2008. p. 446-642.

3. Myerburg RJ, Interian A Jr, Mitrani RM, Kessler KM, Castellanos A. Frequency of sudden cardiac death and profiles of risk. Am J Cardiol. 1997;80:10F-9F.

4. Wilber DJ. Idiopathic ventricular tachycardia. In: Huang SS, Wood MA, editors. Catheter ablation of cardiac arrhythmias. 2nd ed. Philadelphia: Elsevier Saunders; 2010.

5. Aliot EM, Stevenson WG, Almendral-Garrote JM, et al. EHRA/HRS expert consensus on catheter ablation of ventricular arrhythmias: developed in a partnership with the European Heart Rhythm Association (EHRA), a registered branch of the European Society of Cardiology (ESC), and the Heart Rhythm Society (HRS); in collaboration with the American College of Cardiology (ACC) and the American Heart Association (AHA). Heart Rhythm. 2009;6:886-933.

6. Thireau J, Pasquie JL, Martel E, Le Guennec JY, Richard S. New drugs vs. old concepts: a fresh look at antiarrhythmics. Pharmacol Ther. 2011;132:125-45.

7. de Bakker JM, van Capelle FJ, Janse MJ, et al. Reentry as a cause of ventricular tachycardia in patients with chronic ischemic heart disease: electrophysiologic and anatomic correlation. Circulation. 1988;77:589-606.

8. Pogwizd SM, Hoyt RH, Saffitz JE, et al. Reentrant and focal mechanisms underlying ventricular tachycardia in the human heart. Circulation. 1992;86:1872-87.

9. Al-Khatib SM, Granger CB, Huang Y, et al. Sustained ventricular arrhythmias among patients with acute coronary syndromes with no ST-segment elevation: incidence, predictors, and outcomes. Circulation. 2002;106:309-12.

10. Newby KH, Thompson T, Stebbins A, et al. Sustained ventricular arrhythmias in patients receiving thrombolytic therapy: incidence and outcomes. The GUSTO Investigators. Circulation. 1998;98:2567-73.

11. Priori SG, Aliot E, Blomstrom-Lundqvist C, et al. Task Force on Sudden Cardiac Death of the European Society of Cardiology. Eur Heart J. 2001;22:1374-450.

12. Roy D, Marchand E, Theroux P, et al. Long-term reproducibility and significance of provokable ventricular arrhythmias after myocardial infarction. J Am Coll Cardiol. 1986;8:32-9.

13. Callans DJ, Josephson ME. Ventricular tachycardia in patients with coronary artery disease. In: Zipes DP, Jalife J, editors. Cardiac electrophysiology: from cell to bedside. 4th ed. Philadelphia: Elsevier Saunders; 2004. p. 569-74.

14. Epstein AE, Dimarco JP, Ellenbogen KA, et al. 2012 ACCF/AHA/HRS focused update incorporated into the ACCF/AHA/HRS 2008 guidelines for devicebased therapy of cardiac rhythm abnormalities: a report of the American College of Cardiology Foundation/American Heart Association Task Force on Practice Guidelines and the Heart Rhythm Society. Circulation. 2012;60:1297-313.

15. Stecker EC, Chugh SS. Prediction of sudden cardiac death: next steps in pursuit of effective methodology. J Interv Card Electrophysiol. 2011; 31:101-7.

16. Hohnloser SH, Kuck KH, Dorian $\mathrm{P}$, et al. Prophylactic use of an implantable cardioverterdefibrillator after acute myocardial infarction. $\mathrm{N}$ Engl J Med. 2004;351:2481-8.

17. Exner DV, Pinski SL, Wyse DG, et al. Electrical storm presages nonsudden death: the Antiarrhythmics Versus Implantable Defibrillators (AVID) trial. Circulation. 2001;103:2066-71.

18. Patel C, Yan G-X, Kocovic D, Kowey PR. Should catheter ablation be the preferred therapy for reducing ICD shocks?: ventricular tachycardia ablation versus drugs for preventing ICD shocks: role of adjuvant antiarrhythmic drug therapy. Circ Arrhythm Electrophysiol. 2009;2:705-11.

19. Connolly SJ, Dorian P, Roberts RS, et al. Comparison of beta-blockers, amiodarone plus beta-blockers, or sotalol for prevention of shocks from implantable cardioverter defibrillators: the OPTIC Study: a randomized trial. JAMA. 2006;295: 165-71. 
20. Vaughan Williams EM. A classification of antiarrhythmic actions reassessed after a decade of new drugs. J Clin Pharmacol. 1984;24:129-47.

21. Gillis AM. Class I Antiarrhythmic drugs: quinidine, procainamide, disopyramide, lidocaine, mexiletine, flecained and propafenone. In: Zipes DP, Jalife J, editors. Cardiac electrophysiology: from cell to bedside. 4th ed. Philadelphia: Elsevier Saunders; 2004. p. 911-7.

22. Smith TW, Cain ME. Class III antiarrhythmic drugs: amiodarone, ibutilide and sotalol. In: Zipes DP, Jalife J, editors. Cardiac electrophysiology: from cell to bedside. 4th ed. Philadelphia: Elsevier Saunders; 2004. p. 932-41.

23. Singh S, Zoble RG, Yellen L, et al. Efficacy and safety of oral dofetilide in converting to and maintaining sinus rhythm in patients with chronic atrial fibrillation or atrial flutter: the Symptomatic Atrial Fibrillation Investigative Research on Dofetilide (SAFIRE-D) study. Circulation. 2000;102:2385-90.

24. Torp-Pedersen C, Moller M, Bloch-Thomsen PE, et al. Dofetilide in patients with congestive heart failure and left ventricular dysfunction. Danish Investigations of Arrhythmia and Mortality on Dofetilide Study Group. N Engl J Med. 1999;341:857-65.

25. Kober L, Bloch-Thomsen PE, Moller M, et al. Effect of dofetilide in patients with recent myocardial infarction and left-ventricular dysfunction: a randomised trial. Lancet. 2000;356:2052-8.

26. Hohnloser SH, Crijns HJGM, van Eickels M, et al. Effect of dronedarone on cardiovascular events in atrial fibrillation. N Engl J Med. 2009;360:668-78.

27. Singh BN, Connolly SJ, Crijns $\mathrm{HJ}$, et al. Dronedarone for maintenance of sinus rhythm in atrial fibrillation or flutter. N Engl J Med. 2007;357: 987-99.

28. Vizzardi E, D'Aloia A, Quinzani F, et al. A focus on antiarrhythmic properties of ranolazine. J Cardiovasc Pharmacol Ther. 2012;17:353-6.

29. Scirica BM, Morrow DA, Hod H, et al. Effect of ranolazine, an antianginal agent with novel electrophysiological properties, on the incidence of arrhythmias in patients with non ST-segment elevation acute coronary syndrome: results from the Metabolic Efficiency With Ranolazine for Less Ischemia in Non ST-Elevation Acute Coronary Syndrome Thrombolysis in Myocardial Infarction 36 (MERLIN-TIMI 36) randomized controlled trial. Circulation. 2007;116:1647-52.

30. The Cardiac Insufficiency Bisoprolol Study II (CIBISII): a randomised trial. Lancet. 1999;353:9-13.
31. Chen ZM, Pan HC, Chen YP, et al. Early intravenous then oral metoprolol in 45,852 patients with acute myocardial infarction: randomised placebo-controlled trial. Lancet. 2005; 366:1622-32.

32. Pacifico A, Hohnloser SH, Williams JH, et al. Prevention of implantable-defibrillator shocks by treatment with sotalol. D,L-Sotalol Implantable Cardioverter-Defibrillator Study Group. N Engl J Med. 1999;340:1855-62.

33. Waldo AL, Camm AJ, de Ruyter $\mathrm{H}$, et al. Effect of Dsotalol on mortality in patients with left ventricular dysfunction after recent and remote myocardial infarction. The SWORD Investigators. Survival with oral D-sotalol. Lancet. 1996;348:7-12.

34. Boutitie F, Boissel JP, Connolly SJ, et al. Amiodarone interaction with beta-blockers: analysis of the merged EMIAT (European Myocardial Infarct Amiodarone Trial) and CAMIAT (Canadian Amiodarone Myocardial Infarction Trial) databases. The EMIAT and CAMIAT Investigators. Circulation. 1999;99:2268-75.

35. Echt DS, Liebson PR, Mitchell LB, et al. Mortality and morbidity in patients receiving encainide, flecainide, or placebo. The Cardiac Arrhythmia Suppression Trial. N Engl J Med. 1991;324:781-8.

36. Dorian P, Borggrefe M, Al-Khalidi HR, et al. Placebocontrolled, randomized clinical trial of azimilide for prevention of ventricular tachyarrhythmias in patients with an implantable cardioverter defibrillator. Circulation. 2004;110:3646-54.

37. Mason PK, DiMarco JP. New pharmacological agents for arrhythmias. Circ Arrhythm Electrophysiol. 2009;2:588-97.

38. Josephson ME. Evaluation of antiarrhythmic agents. In: Clinical cardiac electrophysiology: techniques and interpretations. 4th ed. Philadelphia: Lippincott Williams \& Wilkins; 2008. p. 643-91.

39. Krum H, Roecker EB, Mohacsi P, et al. Effects of initiating carvedilol in patients with severe chronic heart failure: results from the COPERNICUS Study. JAMA. 2003;289:712-8.

40. Ryden L, Ariniego R, Arnman K, et al. A double-blind trial of metoprolol in acute myocardial infarction. Effects on ventricular tachyarrhythmias. N Engl J Med. 1983;308:614-8.

41. Kaufman ES. Mechanisms and clinical management of inherited channelopathies: long QT syndrome, Brugada syndrome, catecholaminergic polymorphic ventricular tachycardia, and short QT syndrome. Heart Rhythm. 2009;6(Suppl. 8):S51-5. 
42. Hennekens CH, Albert CM, Godfried SL, Gaziano JM, Buring JE. Adjunctive drug therapy of acute myocardial infarction-evidence from clinical trials. N Engl J Med. 1996;335:1660-7.

43. Van Herendael H, Pinter A, Ahmad K, et al. Role of antiarrhythmic drugs in patients with implantable cardioverter defibrillators. Europace. 2010;12: 618-25.

44. Sim I, McDonald KM, Lavori PW, Norbutas CM, Hlatky MA. Quantitative overview of randomized trials of amiodarone to prevent sudden cardiac death. Circulation. 1997;96:2823-9.

45. Bardy GH, Lee KL, Mark DB, et al. Amiodarone or an implantable cardioverter-defibrillator for congestive heart failure. N Engl J Med. 2005;352:225-37.

46. Cairns JA, Connolly SJ, Roberts R, Gent $\mathrm{M}$. Randomised trial of outcome after myocardial infarction in patients with frequent or repetitive ventricular premature depolarisations: CAMIAT, Canadian Amiodarone Myocardial Infarction Arrhythmia Trial Investigators. Lancet. 1997;349: 675-82.

47. Julian DG, Camm AJ, Frangin G, et al. Randomised trial of effect of amiodarone on mortality in patients with left-ventricular dysfunction after recent myocardial infarction: EMIAT. European Myocardial Infarct Amiodarone Trial Investigators. Lancet. 1997;349:667-74.

48. MacNeil DJ. The side effect profile of class III antiarrhythmic drugs: focus on D,L-sotalol. Am J Cardiol. 1997;80(Suppl. 1):90G-8G.

49. Mason JW. A comparison of seven antiarrhythmic drugs in patients with ventricular tachyarrhythmias. Electrophysiologic Study versus Electrocardiographic Monitoring Investigators. N Engl J Med. 1993; 329:452-8.

50. The CASCADE Investigators. Randomized antiarrhythmic drug therapy in survivors of cardiac arrest (the CASCADE Study). Am J Cardiol. 1993;72:280-7.

51. Alexander JH, Granger CB, Sadowski Z, et al. Prophylactic lidocaine use in acute myocardial infarction: incidence and outcomes from two international trials. The GUSTO-I and GUSTO-IIb Investigators. Am Heart J. 1999;137:799-805.

52. Kober L, Torp-Pedersen C, McMurray JJV, et al. Increased mortality after dronedarone therapy for severe heart failure. N Engl J Med. 2008;358: 2678-87.

53. US Food and Drug Administration. FDA drug safety communication: severe liver injury associated with the use of dronedarone (marketed as Multaq). Available at: http://www.fda.gov/drugs/drugsafety/ ucm240011.htm, Accessed Feb 112013.

54. Exposito V, Rodriguez-Entem F, Gonzalez-Enriquez S, Olalla JJ. Dronedarone for recurrent ventricular tachycardia: a real alternative? Indian Pacing Electrophysiol J. 2012;12:73-6.

55. Fink A, Duray GZ, Hohnloser SH. A patient with recurrent atrial fibrillation and monomorphic ventricular tachycardia treated successfully with dronedarone. Europace. 2011;13:284-5.

56. Shaaraoui M, Freudenberger R, Levin V, Marchlinski FE. Suppression of ventricular tachycardia with dronedarone: a case report. J Cardiovasc Electrophysiol. 2011;22:201-2.

57. Connolly SJ, Camm AJ, Halperin JL, et al. Dronedarone in high-risk permanent atrial fibrillation. N Engl J Med. 2011;365:2268-76.

58. Carmeliet E. Voltage- and time-dependent block of the delayed $\mathrm{K}+$ current in cardiac myocytes by dofetilide. J Pharmacol Exp Ther. 1992;262:809-17.

59. Boriani G, Lubinski A, Capucci A, et al. A multicentre, double-blind randomized crossover comparative study on the efficacy and safety of dofetilide vs sotalol in patients with inducible sustained ventricular tachycardia and ischaemic heart disease. Eur Heart J. 2001;22:2180-91.

60. Baquero GA, Banchs JE, Depalma S, et al. Dofetilide reduces the frequency of ventricular arrhythmias and implantable cardioverter defibrillator therapies. J Cardiovasc Electrophysiol. 2012;23:296-301.

61. Frommeyer G, Kaiser D, Uphaus T, et al. Effect of ranolazine on ventricular repolarization in class III antiarrhythmic drug-treated rabbits. Heart Rhythm. 2012;9:2051-8.

62. Verrier RL, Pagotto VP, Kanas AF, et al. Low doses of ranolazine and dronedarone in combination exert potent protection against atrial fibrillation and vulnerability to ventricular arrhythmias during acute myocardial ischemia. Heart Rhythm. 2012;1:121-7.

63. Kaliebe JW, Murdock DK. Suppression of nonsustained ventricular tachycardia with ranolazine: a case report. WMJ. 2009;108:373-5.

64. Bunch TJ, Mahapatra S, Murdock D, et al. Ranolazine reduces ventricular tachycardia burden and ICD shocks in patients with drug-refractory ICD shocks. Pacing Clin Electrophysiol. 2011;34: 1600-6.

65. Sicouri S, Burashnikov A, Belardinelli L, Antzelevitch C. Synergistic electrophysiologic and 
antiarrhythmic effects of the combination of ranolazine and chronic amiodarone in canine atria. Circ Arrhythm Electrophysiol. 2010;3:88-95.

66. Karwatowska-Prokopczuk E, Wang W, Cheng ML, et al. The risk of sudden cardiac death in patients with non-ST elevation acute coronary syndrome and prolonged QTc interval: effect of ranolazine. Europace. 2012 [Epub ahead of print].

67. Camm AJ, Pratt CM, Schwartz PJ, et al. Mortality in patients after a recent myocardial infarction: a randomized, placebo-controlled trial of azimilide using heart rate variability for risk stratification. Circulation. 2004;109:990-6.

68. Kowey PR, Crijns HJ, Aliot EM, et al. Efficacy and safety of celivarone, with amiodarone as calibrator, in patients with an implantable cardioverterdefibrillator for prevention of implantable cardioverter-defibrillator interventions or death: the ALPHEE study. Circulation. 2011;124:2649-60.

69. Gojkovic O, Aliot EM, Capucci A, et al. Celivarone in patients with an implantable cardioverterdefibrillator: adjunctive therapy for the reduction of ventricular arrhythmia-triggered implantable cardioverter-defibrillator interventions. Heart Rhythm. 2012;9(217-24):e2.

70. Hong RA, Rivera KK, Jittirat A, Choi JJ. Flecainide suppresses defibrillator-induced storming in catecholaminergic polymorphic ventricular tachycardia. Pacing Clin Electrophysiol. 2012;35: 794-7.
71. Sacherer M, Sedej S, Wakula P, et al. JTV519 (K201) reduces sarcoplasmic reticulum $\mathrm{Ca}(2)(+)$ leak and improves diastolic function in vitro in murine and human non-failing myocardium. Br J Pharmacol. 2012;167:493-504.

72. Degrande S, Nixon D, Koval O, et al. CaMKII inhibition rescues proarrhythmic phenotypes in the model of human ankyrin-B syndrome. Heart Rhythm. 2012;9:2034-41.

73. Wit AL, Duffy HS. Drug development for treatment of cardiac arrhythmias: targeting the gap junctions. Am J Physiol Heart Circ Physiol. 2008;294:H16-8.

74. Kjolbye AL, Dikshteyn M, Eloff BC, Deschenes I, Rosenbaum DS. Maintenance of intercellular coupling by the antiarrhythmic peptide rotigaptide suppresses arrhythmogenic discordant alternans. Am J Physiol Heart Circ Physiol. 2008;294:H41-9.

75. Antoons G, Sipido KR. Targeting calcium handling in arrhythmias. Europace. 2008;10:1364-9.

76. Billman GE. The cardiac sarcolemmal ATP-sensitive potassium channel as a novel target for antiarrhythmic therapy. Pharmacol Ther. 2008;120: 54-70.

77. Englert HC, Gerlach U, Goegelein $\mathrm{H}$, et al. Cardioselective K(ATP) channel blockers derived from a new series of $\mathrm{m}$-anisamidoethylbenzenesulfonylthioureas. J Med Chem. 2001;44:1085-98. 